\title{
Tuning gaps and phases of a two-subband system in a quantizing magnetic field
}

\author{
V. M. Apalkov and M. E. Portnoi* \\ School of Physics, University of Exeter, Stocker Road, Exeter EX4 4QL, United Kingdom
}

(Received 21 July 2001; published 11 March 2002)

\begin{abstract}
In this work we study the properties of a two-subband quasi-two-dimensional electron system in a strong magnetic field when the electron filling factor is equal to 4 . When the cyclotron energy is close to the intersubband splitting the system can be mapped onto a four-level electron system with an effective filling factor of 2. The ground state is either a ferromagnetic state or a spin-singlet state, depending on the values of the interlevel splitting and Zeeman energy. The boundaries between these phases are strongly influenced by the interelectron interaction. A significant exchange-mediated enhancement of the excitation gap results in the suppression of the electron-phonon interaction. The rate of absorption of nonequilibrium phonons is calculated as a function of Zeeman energy and intersubband splitting. The phonon absorption rate has two peaks as a function of intersubband splitting and has a steplike structure as a function of Zeeman energy.
\end{abstract}

DOI: 10.1103/PhysRevB.65.125310

PACS number(s): 73.43.Nq, 73.43.Lp, 63.20.Kr

During the last two decades the behavior of a twodimensional (2D) electron system in a quantizing magnetic field attracted a lot of theoretical and experimental interest. The peculiar properties of this system result from its effective zero dimensionality, because the magnetic field normal to the 2D layer quenches the electron kinetic energy to a constant. As a result the Hamiltonian of the system consists of the interaction part only, and the system undergoes a transition into an incompressible state at certain magnetic field values. The formation of the incompressible states results in the fractional quantum Hall effect-one of the most interesting phenomena discovered in strongly correlated electron systems. ${ }^{1-3}$ The only typical energy of the system is the characteristic electron-electron Coulomb interaction energy, which is of the order of $\varepsilon_{C}=e^{2} / \kappa l$, where $l$ is the magnetic length and $\kappa$ is the dielectric constant. The introduction of a new degree of freedom into this system results in new interesting phenomena when the characteristic energy of the new degree of freedom becomes smaller than the Coulomb energy $\varepsilon_{C}$. The electron spin is one of the possible degrees of freedom. If the Zeeman energy is small enough, then the transition from the spin-polarized to spin-unpolarized ground state occurs at electron filling factor $\nu_{0}=2 / m$, where $m$ is odd. ${ }^{4}$ At filling factor $\nu_{0}=1 / \mathrm{m}$ a new type of charged topological excitations, Skyrmions, appears.

One can also introduce the degree of freedom in the direction normal to the $2 \mathrm{D}$ layer. This can be realized in a double-layer system. In this case the phase diagram is driven by the interplay between the electron-electron Coulomb energy, the tunneling energy between two layers, and the Zeeman energy. It was found that at $\nu_{0}=2$ the double-layer system can be found in three different phases: fully spinpolarized ferromagnetic state, spin-singlet state, and canted antiferromagnetic state. ${ }^{5}$

In this paper we consider a system which is similar to a double-layer system but has a different geometry, which provides more experimental possibilities to change the parameters of the system. Namely, we study a single heterojunction in which the second degree of freedom is introduced by the size quantization in the direction perpendicular to the $2 \mathrm{D}$ layer. If the electron filling factor is greater than 2 and the energy $\Delta=\Delta_{12}-\hbar \omega_{c}$ is smaller than the Coulomb energy, then the second subband has to be taken into account. Here $\hbar \omega_{c}$ is the cyclotron energy and $\Delta_{12}$ is the intersubband splitting. In this case the second Landau level (Landau level number $n=1$ ) of the first subband is close in energy to the first Landau level $(n=0)$ of the second subband. Since the intersubband splitting $\Delta_{12}$ has a weak dependence on the magnetic field, the separation $\Delta$ can be changed by changing the magnetic field $B$. The influence of the second subband on the optical properties of the magnetically quantized quasi-2D systems was observed in different types of experiments, e.g., in extrinsic radiative recombination magnetospectroscopy ${ }^{6}$ and in the optically detected cyclotron resonance in a tilted magnetic field. ${ }^{7}$ It was proposed in Ref. 8 that the intersection between the levels can be also observed in the magnetic field dependence of the phonon-mediated conductivity of the system. At filling factor slightly greater than 2 , the dissipative conductivity should reveal a double-peak structure as a function of magnetic field. A similar double-peak structure should be observed in the other phonon spectroscopy experiments. $^{9-11}$ With increasing electron density the repulsion between the levels due to electron-electron exchange interactions opens a gap in the interlevel excitation spectra. As a result the double-peak structure transforms into a single-peak one. ${ }^{12}$

In what follows we study the properties of the twosubband system when the electron filling factor is equal to 4, $\nu_{0}=4$. We assume that the Coulomb energy is much smaller than the cyclotron energy. In this case the completely occupied lowest Landau level of the first subband can be considered as a nondynamical background. We will be interested only in a subsystem consisting of the second Landau level $(n=1)$ of the first subband and the first Landau level $(n$ $=0$ ) of the second subband, the spin degeneracy of both levels being lifted by the Zeeman splitting $\Delta_{z}$. This fourlevel subsystem is schematically shown in Fig. 1 . For $\nu_{0}$ $=4$ the effective filling factor $\nu$ of this subsystem is equal to 2. Under this mapping the system becomes similar to a double-layer system with the total filling factor $\nu=2$ and for its analysis we follow the method of Ref. 5.

For noninteracting electrons we can distinguish three 
cases: (1) if $\Delta>\Delta_{z}$, states " 1 " and " 2 " are occupied, and the ground state is a spin-singlet state; (2) if $-\Delta_{z}<\Delta$ $<\Delta_{z}$, states " 1 " and " 3 " are occupied, and the ground state is a ferromagnetic state; (3) if $\Delta<-\Delta_{z}$, states " 3 " and " 4 " are occupied, and the ground state is again a spin-singlet state. The boundaries between these phases are given by the equations $\Delta=\Delta_{z}$ and $\Delta=-\Delta_{z}$. When the electron-electron interaction is taken into account the transitions between spinsinglet and ferromagnetic states can occur through a new phase-for example, through a canted antiferromagnetic phase. ${ }^{5}$ Our calculations show that in the present system there is no intermediate phase and the transitions from the singlet to the ferromagnetic state are sharp, like in the noninteracting case.

In what follows we use the Coulomb energy $\varepsilon_{C}$ as the unit of energy and magnetic length $l$ as the unit of length. In the Landau gauge with the vector potential $\vec{A}=(0, B x, 0)$ the eigenstates of a single-electron Hamiltonian are characterized by the Landau level number $n ; y$ component of the momentum, $k_{y}$; the electron subband number $\mu=1$ or $\mu$ $=2$; and the $z$ projection of the electron spin, $\sigma=2 S_{z}= \pm 1$ :

$$
\psi_{n, k_{y}, \mu, \sigma}(x, y, z)=\chi_{\mu}(z) \xi_{\sigma} \frac{e^{i k_{y} y}}{\sqrt{L_{y}}} \phi_{n}\left(x-k_{y}\right)
$$

where $\chi_{\mu}(z)$ is the envelope wave function of the $\mu$ th subband; $\xi_{\sigma}$ is the spin part of the wave function, and $\phi_{n}(x)$ is the $n$th harmonic oscillator function.

The Hamiltonian of the interacting electron system is

$$
\begin{aligned}
H= & \sum_{n \mu \sigma k_{y}} \hbar \omega_{c}\left(n+\frac{1}{2}\right) C_{n k_{y} \mu \sigma}^{\dagger} C_{n k_{y} \mu \sigma}+\frac{\Delta}{2} \sum_{n \mu \sigma k_{y}}(2 \mu-3) \\
& \times C_{n k_{y} \mu \sigma}^{\dagger} C_{n k_{y} \mu \sigma}+\frac{\Delta_{z}}{2} \sum_{n \mu \sigma k_{y}} \sigma C_{n k_{y} \mu \sigma}^{\dagger} C_{n k_{y} \mu \sigma} \\
& +\frac{1}{2} \sum_{\{n\}} \sum_{\sigma_{1} \sigma_{2}} \sum_{\{\mu\}} \sum_{q_{x}, q_{y}} \widetilde{V}_{\left(\mu_{1} \mu_{4} \mu_{3} \mu_{2}\right)}^{\left(n_{1} n_{4} n_{3} n_{2}\right)}(\hat{q}) \times \sum_{k_{1}, k_{2}} e^{i q_{x}\left(k_{1}-k_{2}\right)} \\
& \times C_{n_{1}, k_{1}+q_{y}, \mu_{1} \sigma_{1}}^{\dagger} C_{n_{2}, k_{2}, \mu_{2} \sigma_{2}}^{\dagger} C_{n_{3}, k_{2}+q_{y}, \mu_{3} \sigma_{2}} C_{n_{4}, k_{1}, \mu_{4} \sigma_{1}},
\end{aligned}
$$

where $C_{n, k, \mu \sigma}^{\dagger}$ and $C_{n, k, \mu \sigma}$ are the creation and annihilation operators of the electron in the state $\psi_{n, k_{y}, \mu, \sigma}$. In Eq. (2) we use the notation

$$
\begin{aligned}
\widetilde{V}_{\left(i_{1} i_{4} i_{3} i_{2}\right)}^{\left(n_{1} n_{4} n_{3} n_{2}\right)}(\hat{q})= & \frac{1}{q} F_{i_{1} i_{4} i_{3} i_{2}}(q) G_{n_{1} n_{4}}(\hat{q}) G_{n_{3} n_{2}}\left(\hat{q}^{*}\right) \\
& \times \exp \left(-\frac{q^{2}}{2}\right)
\end{aligned}
$$

where $^{13}$

$$
G_{n_{1} n_{2}}(\hat{q})=\left(\frac{n_{1} !}{n_{2} !}\right)^{1 / 2}\left(\frac{-i \hat{q}}{\sqrt{2}}\right)^{n_{2}-n_{1}} L_{n_{1}}^{n_{2}-n_{1}}\left(\frac{q^{2}}{2}\right)
$$

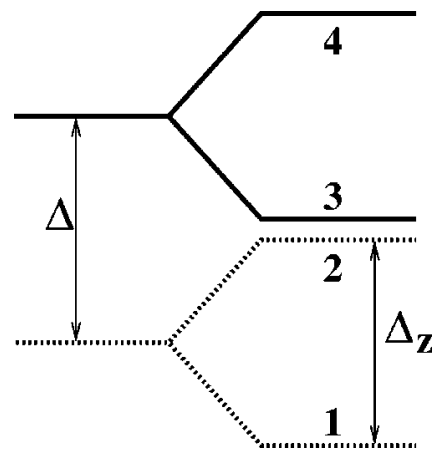

FIG. 1. The single-electron energy levels of the two-subband system. Levels " 1 " and " 2 " belong to the second Landau level $(n=1)$ of the first subband with spins $S_{z}=1 / 2$ and $S_{z}=-1 / 2$, respectively. Levels " 3 " and " 4 " belong to the first Landau level $(n=0)$ of the second subband with spins $S_{z}=1 / 2$ and $S_{z}=-1 / 2$, respectively. The splitting $\Delta$ is the interlevel splitting, $\Delta_{z}$ is the Zeeman energy.

$\hat{q}=q_{x}+i q_{y}, q=|\hat{q}|$, and $L_{n}^{m}$ is a generalized Laguerre polynomial. The modification of the Coulomb interaction due to the finite extent of the electron wave functions in the $z$ direction is given by

$$
\begin{aligned}
F_{i_{1} i_{4} i_{2} i_{3}}(q)= & \int_{0}^{\infty} \int_{0}^{\infty} d z_{1} d z_{2} e^{-q\left|z_{1}-z_{2}\right|} \\
& \times \chi_{i_{1}}\left(z_{1}\right) \chi_{i_{4}}\left(z_{1}\right) \chi_{i_{2}}\left(z_{2}\right) \chi_{i_{3}}\left(z_{2}\right) .
\end{aligned}
$$

We use the Fang-Howard approximation ${ }^{14}$ for the envelope wave functions of the electrons in the first and second subbands:

$$
\begin{gathered}
\chi_{1}(z)=\sqrt{\frac{b^{3}}{2}} z \exp \left(-\frac{1}{2} b z\right), \\
\chi_{2}(z)=\sqrt{\frac{b^{5}}{6}} z\left(z-\frac{3}{b}\right) \exp \left(-\frac{1}{2} b z\right) .
\end{gathered}
$$

We follow the standard Hartree-Fock method, assuming a nonzero average of $\left\langle C_{k_{1}, \mu_{1} \sigma_{1}}^{\dagger} C_{k_{2}, \mu_{2} \sigma_{2}}\right\rangle$ over the ground state. The specific feature of the given problem, which differs it from the double-layer system, is that a nonzero paring in Eq. (2) can occur between states with different values of $k_{y}$, because in our case the two-level system is formed by the different Landau levels. ${ }^{15}$ For each value of $k_{y}=k$ we introduce the new wave functions, which are the eigenfunctions of the Hartree-Fock Hamiltonian. The creation and annihilation operators corresponding to the new wave functions are $a_{k, i}^{\dagger}, a_{k, i}$, where $i=1,2,3$, or 4 . These functions are related to the original ones by the matrix $\gamma_{i, y}$ :

$$
\begin{gathered}
C_{k, 1,-1}=\sum_{i=1}^{4} \gamma_{1 i} a_{k, i}, \\
C_{k, 1,1}=\sum_{i=1}^{4} \gamma_{2 i} a_{k, i},
\end{gathered}
$$




$$
\begin{gathered}
C_{k+Q, 2,-1}=\sum_{i=1}^{4} \gamma_{3 i} a_{k, i}, \\
C_{k+Q, 2,1}=\sum_{i=1}^{4} \gamma_{4 i} a_{k, i} .
\end{gathered}
$$

The average of the introduced functions over the ground state is $\left\langle a_{k, i}^{\dagger} a_{k, j}\right\rangle=\delta_{i j}\left(\delta_{i 1}+\delta_{i 2}\right)$. This means that only the states with the lowest energies ( $i=1$ and $i=2)$ are occupied. Substituting Eq. (4) into the Hamiltonian (2) we obtain the Hartree-Fock Hamiltonian in the form of a $4 \times 4$ matrix. In the basis $\left(C_{k, 1,-1}, C_{k, 1,1}, C_{k+Q, 2,-1}, C_{k+Q, 2,1}\right)$ the elements of this matrix are

$$
\begin{gathered}
H_{11}^{H F}=-\frac{1}{2}\left(\Delta+\Delta_{z}\right)-\beta_{11} \epsilon_{11}^{11}-\beta_{33} \epsilon_{12}^{01}, \\
H_{22}^{H F}=-\frac{1}{2}\left(\Delta-\Delta_{z}\right)-\beta_{22} \epsilon_{11}^{11}-\beta_{44} \epsilon_{12}^{01}, \\
H_{33}^{H F}=\frac{1}{2}\left(\Delta-\Delta_{z}\right)-\beta_{33} \epsilon_{22}^{00}-\beta_{11} \epsilon_{12}^{01}, \\
H_{44}^{H F}=\frac{1}{2}\left(\Delta+\Delta_{z}\right)-\beta_{44} \epsilon^{00} 22-\beta_{44} \epsilon_{12}^{01}, \\
H_{12}^{H F}=-\beta_{12} \epsilon_{11}^{11}-\left(\beta_{23}-\beta_{14}\right) V_{(2111)}^{(0100)}(Q)-\beta_{34} \epsilon_{12}^{01}, \\
H_{13}^{H F}=-\beta_{13}\left[V_{(1122)}^{(0011)}(0)+V_{(1212)}^{(0101)}(Q)\right], \\
H_{14}^{H F}=-\beta_{23} V_{(1212)}^{(0101)}(Q)-\beta_{14} V_{(1122)}^{(0011)}(0), \\
H_{23}^{H F}=-\beta_{14} V_{(1212)}^{(0101)}(Q)-\beta_{23} V_{(1122)}^{(0011)}(0), \\
H_{24}^{H F}=-\beta_{24}\left[V_{(1122)}^{(0011)}(0)+V_{(1212)}^{(0101)}(Q)\right], \\
H_{34}^{H F}=-\beta_{34} \epsilon_{22}^{00}-\left(\beta_{23}-\beta_{14}\right) V_{(1222)}^{(0111)}(Q)-\beta_{12} \epsilon_{12}^{01},
\end{gathered}
$$

where $\beta_{i j}=\gamma_{i 1} \gamma_{j 1}+\gamma_{i 2} \gamma_{j 2}$,

$$
\begin{aligned}
V_{\left(j_{1} j_{2} j_{3} j_{4}\right)}^{\left(i_{1} i_{2} i_{3} i_{4}\right)}(q)= & \int_{0}^{\infty} d k F_{j_{1} j_{2} j_{3} j_{4}}(k)\left|G_{i_{1} i_{2}}(k)\right|\left|G_{i_{3} i_{4}}(k)\right| \\
& \times J_{\left|i_{1}+i_{3}-i_{2}-i_{4}\right|}(k q) \exp \left(-\frac{k^{2}}{2}\right), \\
& \epsilon_{j_{1} j_{2}}^{i_{1} i_{2}}=V_{\left(j_{1} j_{2} j_{1} j_{2}\right)}^{\left(i_{1} i_{2} i_{1} i_{2}\right)}(0),
\end{aligned}
$$

$J_{m}$ is the Bessel function of $m$ th order, and $\epsilon_{j_{1} j_{2}}^{i_{1} i_{2}}$ is the exchange energy of an electron in the $i_{1}$ th Landau level of the $j_{1}$ th subband interacting with electrons of the same spin in the filled $i_{2}$ th Landau level of the $j_{2}$ th subband.

Taking into account that the eigenvectors of the HartreeFock matrix $a_{k, i}$ are related to $C_{k, \mu, \sigma}$ by the matrix $\gamma_{i j}$ [Eq. (4)], we obtain a self-consistent system of equations for $\gamma_{i j}$. The parameter $Q$ can be found by minimizing the HartreeFock energy. We have found the solution of the system of

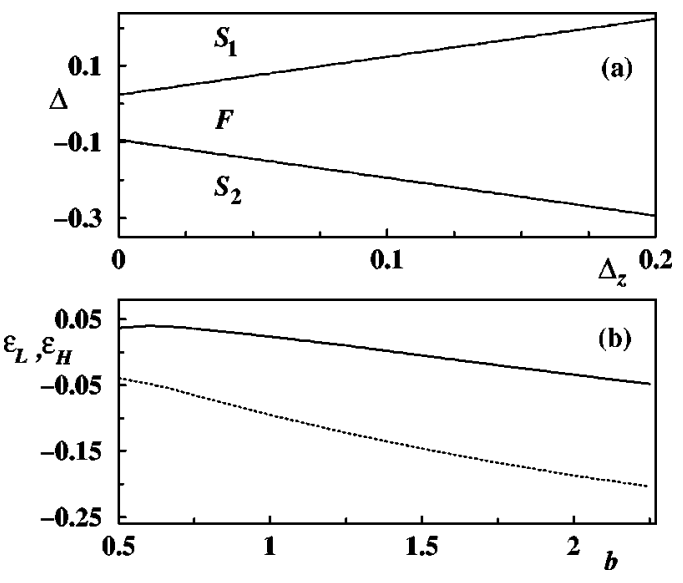

FIG. 2. (a) The phase diagram of the four-level system for $b$ $=1$. The phases $S_{1}$ and $S_{2}$ are spin-singlet phases; the phase $F$ is a ferromagnetic phase. (b) The parameters $\epsilon_{H}$ and $\epsilon_{L}$ [Eqs. (6) and (7)] of the phase diagram are plotted as the functions of $b$. All energies are in units of $\varepsilon_{C} ; b$ is in units of $1 / l$.

equations for $\gamma_{i j}$ numerically. For all values of $\Delta$ and $\Delta_{z}$ the ground state of the system is one of the noninteracting phases (spin singlet or ferromagnetic), described above. The transitions between the phases are sharp. The interaction modifies the phase boundaries only:

$$
\begin{gathered}
\Delta=\Delta_{z}+\frac{1}{2}\left(\epsilon_{22}^{00}-\epsilon_{11}^{11}\right)+\epsilon_{12}^{00}-\epsilon_{11}^{01}+\epsilon_{12}^{01}=\Delta_{z}+\epsilon_{H}=\Delta_{H}, \\
\Delta=-\Delta_{z}+\frac{1}{2}\left(\epsilon_{22}^{00}-\epsilon_{11}^{11}\right)+\epsilon_{12}^{00}-\epsilon_{11}^{01}-\epsilon_{12}^{01}=-\Delta_{z}+\epsilon_{L}=\Delta_{L},
\end{gathered}
$$

where $\Delta_{H}$ and $\Delta_{L}$ denote the higher and lower values of splitting $\Delta$ at the boundary of the ferromagnetic phase; see Fig. 2(a). In Fig. 2(a) the phase diagram is shown for $b=1$. The phases $S_{1}$ and $S_{2}$ are the spin-singlet phases and the phase $F$ is the ferromagnetic phase. The single-electron states " 1 " and " 2 ," " 1 " and " 3 ," and " 3 " and "4" (Fig. 1) are occupied in the phases $S_{1}, F$, and $S_{2}$, respectively. The electron-electron interaction results in the splitting of the boundaries between different phases, even when the Zeeman energy is equal to zero. For $\Delta_{z}=0$ the splitting is equal to $\epsilon_{H}-\epsilon_{L}=2 \epsilon_{12}^{01}$, where $\epsilon_{H}$ and $\epsilon_{L}$ are introduced in Eqs. (6) and (7). In Fig. 2(b) the values of the lower $\left(\epsilon_{L}\right)$ and upper $\left(\epsilon_{H}\right)$ phase boundaries at $\Delta_{z}=0$ are shown as a function of the Fang-Howard parameter $b$, where $1 / b$ is proportional to the characteristic width of the heterojunction in units of magnetic length. Both values $\epsilon_{H}$ and $\epsilon_{L}$ decrease with increasing $b$.

We are mostly interested in the case when the intersubband splitting is close to the cyclotron energy. This gives the restriction of the values of the parameter $b$. This parameter should be close to the inverse magnetic length. However, the exact relation between $b$ and $l$ for $\Delta=0$ depends on the 
actual value of $b$. Therefore we found it appropriate to calculate the $b$ dependences of the main parameters of the system.

The two-subband system can be studied experimentally by changing the magnetic field when keeping the fixed value of the filling factor. If the magnetic field $\vec{B}$ is normal to the heterojunction, then both $-\Delta$ and $\Delta_{z}$ increase linearly with increasing $B$. In this case only the transition from the phase $S_{1}$ to the phase $F$ can be observed. Another possibility is to make the experiments in the tilted magnetic field with the fixed value of its normal component. In this case $\Delta$ is a constant, because it is proportional to the normal component of the magnetic field, but $\Delta_{z}$ changes linearly with the total magnetic field. Depending on the initial value of $\Delta$, one should observe a transition from $S_{1}$ to $F$ or from $S_{2}$ to $F$ phases. However, there is a finite region of $\Delta$ in which no transitions can be found and the system is always in the phase $F$. This region has a nonzero range even for zero $\Delta_{z}$ when its width is $2 \epsilon_{12}^{01}$.

Now we shall discuss the possibility to detect the different phases by acoustic-phonon spectroscopy. ${ }^{9-11}$ We consider the absorption of nonequilibrium phonons by the discussed quasi-2D system. The electron-phonon interaction is proportional to the electron density operator. ${ }^{16}$ During the act of phonon absorption the electron system undergoes a transition from the ground state to the excited state, corresponding to the density fluctuations. When the system is in the ferromagnetic phase $(F)$, there are spin reversal excitations only. In this case phonon absorption is forbidden. The collective excitation spectra in the singlet phases $S_{1}$ and $S_{2}$ can be found

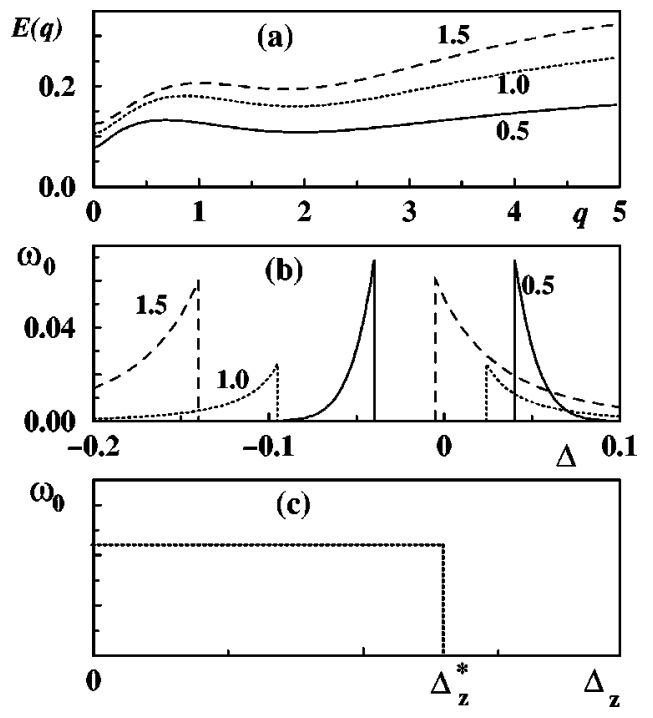

FIG. 3. (a) The excitation dispersion $E(q)$ [Eq. (10)] for $\delta \Delta$ $=0=\Delta_{z}$. The numbers near the lines show the values of the parameter $b$, where $b$ and $q$ are in units of $1 / l ; E(q)$ is in units of $\varepsilon_{C}$. (b) The phonon absorption rate $\omega_{0}$ as a function of $\Delta$ for $\Delta_{z}=0$. Solid, dotted, and dashed lines are for $b=0.5,1.0$, and 1.5, respectively. The data for $b=1.0$ and 1.5 are multiplied by 10 . The absorption rate $\omega_{0}$ is in units of $10^{10} \mathrm{~s}^{-1} ; \Delta$ is in units of $\varepsilon_{C}$. (c) Phonon absorption rate $\omega_{0}$ as a function of $\Delta_{z}$ for constant $\Delta$. Here $\omega_{0}$ and $\Delta_{z}$ are in arbitrary units. The critical Zeeman energy $\Delta_{z}^{*}$ corresponds to the $S_{1} \rightarrow F$ or $S_{2} \rightarrow F$ phase transition. from the poles of the density-density correlation function, ${ }^{13}$ which are given by the expressions

$$
E(q)=\Delta+2 \widetilde{V}_{1212}^{0101}(q)-V_{1122}^{0011}(q)-\epsilon_{12}^{01}-\epsilon_{12}^{00}+\epsilon_{11}^{11}+\epsilon_{11}^{01}
$$

for the phase $S_{1}$ and

$$
E(q)=-\Delta+2 \widetilde{V}_{1212}^{0101}(q)-V_{1122}^{0011}(q)-\epsilon_{12}^{01}-\epsilon_{11}^{01}+\epsilon_{22}^{00}+\epsilon_{12}^{00}
$$

for the phase $S_{2}$, where Eqs. (3) and (5) were used. One can rewrite Eqs. (8) and (9) in the form, which can describe both cases simultaneously

$$
E(q)=|\delta \Delta|+\Delta_{z}+2 \widetilde{V}_{1212}^{0101}(q)-V_{1122}^{0011}(q)+\frac{1}{2}\left(\epsilon_{22}^{00}+\epsilon_{11}^{11}\right),
$$

where $\delta \Delta=\Delta-\Delta_{H}$ for the phase $S_{1}$ and $\delta \Delta=-\Delta+\Delta_{L}$ for the phase $S_{2}$.

The energy spectra $E(q)$ are shown in Fig. 3(a) for $\delta \Delta$ $=0$ and $\Delta_{z}=0$, for several different values of the parameter $b$. One can see that there is a finite gap for all values of momentum $q$. This means that a gap exists for any value of $\Delta$ and $\Delta_{z}$. With increasing the spreading of the electron wave function in the $z$ direction (decreasing $b$ ) the gap becomes smaller, which results from the decreasing of the effective $2 \mathrm{D}$ electron-electron interaction. The existence of an interaction-induced finite excitation gap in the two-level system for any value of the interlevel splitting was demonstrated for a system with filling factor $\nu=1$ in Ref. 12. The effect of the strong renormalization of the excitation energy due to the interelectron interaction is also known for spin excitations as an interaction enhancement of the $g$ factor in $2 \mathrm{D}$ systems. $^{18-21}$

The electron-phonon interaction Hamiltonian has the form

$$
H_{e-p h}=-\sum_{j, \vec{Q}} \frac{M_{j}(\vec{Q})}{\sqrt{V}} Z\left(q_{z}\right)\left[\rho(\vec{q}) \hat{d}_{j}^{\dagger}(\vec{Q})+\rho(-\vec{q}) \hat{d}_{j}(\vec{Q})\right]
$$

where the isotropic Debye approximation is used with the linear dependence of the phonon frequency on the wave vector: $\omega_{j}(K)=s_{j} K, s_{j}$ being speed of sound; $j$ labels the phonon modes, $j=1$ for the longitudinal mode and $j=2,3$ for the two transverse modes. In Eq. (11) we labeled the threedimensional (3D) phonon wave vector by the capital letter $\vec{Q}$ and its projections by the corresponding small letters $\vec{Q}$ $=\left(\vec{q}, q_{z}\right)$. The creation operator of a phonon in the $j$ th mode is denoted as $\hat{d}_{j}^{\dagger}, V$ is a normalization volume, $\rho(\vec{q})$ is the two-dimensional electron density operator, and $M_{j}(\vec{Q})$ is the matrix element of the electron-phonon interaction, which is determined by the deformation potential and piezoelectric coupling: ${ }^{17}$ 


$$
\begin{aligned}
M_{j}(\vec{Q})= & \sqrt{\frac{\hbar}{2 \rho_{0} s}}\left[-\beta \frac{Q_{x} Q_{y} \xi_{j, z}+Q_{y} Q_{z} \xi_{j, x}+Q_{z} Q_{x} \xi_{j, y}}{Q^{2}}\right. \\
& \left.-i \Xi_{0}\left(\vec{\xi}_{j} \cdot \vec{Q}\right)\right],
\end{aligned}
$$

where $\rho_{0}$ is the mass density, $\beta$ and $\Xi_{0}$ are the parameters of the piezoelectric and deformation potential couplings, and $\vec{\xi}_{j}$ is the polarization vector of the $j$ th phonon mode. The parameters of GaAs are used in our calculations.

Because the phonon-assisted transitions are only allowed between the different subbands, the form factor $Z\left(q_{z}\right)$ is

$$
Z\left(q_{z}\right)=\int d z e^{i q_{z} z} \chi_{1}(z) \chi_{2}(z)
$$

At low temperature $k_{B} T \ll E(q)$, the rate of absorption of nonequilibrium phonons can be found from the expression $^{12,17}$

$$
\begin{aligned}
\omega_{a b s}= & \frac{2 \pi}{\hbar} \sum_{j} \int \frac{d \vec{Q}}{(2 \pi)^{3}} \delta\left(\mathbf{E}(q)-s_{j} Q\right) \mathbf{n}_{j}(\vec{Q}) \\
& \times\left|M_{j}(\vec{Q}) Z\left(q_{z}\right)\right|^{2} R_{01}(q),
\end{aligned}
$$

where $R_{01}(q)=\left(q^{2} / 2\right) \exp \left(-q^{2} / 2\right)$, and $n_{j}(\vec{Q})$ is the phonon distribution function.

One can see how the appearance of the excitation gap suppresses the phonon absorption. The phonon absorption process requires the conservation of energy and in-plane momentum, $s_{j} \sqrt{q^{2}+q_{z}^{2}}=E(q)$, where the characteristic excitation gap $E(q)$ is greater than 0.1 for $b \sim 1$ [see Fig. 3(a)]. The GaAs speed of sound in units of $l \epsilon_{C} / \hbar$ is about 0.03 for longitudinal and 0.02 for transverse phonons. Therefore, a large value of $\sqrt{q^{2}+q_{z}^{2}}$ is required to satisfy the energy conservation law. However, the factors $R_{01}(q)$ and $Z\left(q_{z}\right)$ in Eq. (14) make the phonon absorption to be small at $q>1$ or $q_{z}$ $>b$. Thus, the phonon absorption becomes strongly suppressed compared to the noninteracting case, in which the excitation energy $\Delta$ is allowed to have any value. For the noninteracting electrons the absorption rate has two maxima at $|\Delta| \sim \hbar s_{j} / l .^{8}$
From Eq. (14) we calculate the normalized phonon absorption rate $\omega_{0}=\omega_{a b s} / n\left(E\left(q_{0}\right)\right)$, where $q_{0}$ is a characteristic momentum; here we use $q_{0}=1$. In Fig. 3(b) the rate $\omega_{0}$ is shown as a function of $\Delta$ for $\Delta_{z}=0$ and different values of $b$. The absorption rate has a two-peak structure. The gap between the peaks corresponds to the phase $F$ of the system, in which the phonons cannot be absorbed. The absorption rates for $b=1$ and $b=1.5$ are multiplied by 10 . With increasing $b$ the collective excitation gap increases [see Fig. 3(a)], which results in a rapid decrease of the absorption rate. One can see this tendency for $b=0.5$ and $b=1$. However, with increasing $b$, phonons with larger values of $q_{z}$ can be absorbed, which tends to increase the absorption rate. The latter effect becomes more pronounced for large $b$ when the excitation gap has a weaker dependence on $b$ [see Fig. 3(a)]. The competition between these two effects results in a slight increase of the absorption rate for $b=1.5$.

In tilted magnetic field experiments, when the Zeeman energy $\Delta_{z}$ is changed while the interlevel splitting $\Delta$ is kept fixed, the absorption rate remains constant. For the singlet phase $S_{1}$ or $S_{2}$ the absorption rate is given by Eqs. (8), (9), and (14) and for the ferromagnetic phase $F$ the absorption rate is zero. The dependence of $\omega_{0}$ on $\Delta_{z}$ is shown schematically in Fig. 3(c), where the critical value of the Zeeman splitting, $\Delta_{z}^{*}$, corresponds to the $S_{1}-F$ or $S_{2}-F$ phase transition.

In conclusion, we considered a two-subband electron system with total filling factor 4 . The system was mapped onto the four-level electron system with effective filling factor 2. The separation between the levels belonging to the different subbands is proportional to the cyclotron energy and can be changed by changing the magnetic field. The system exists in one of the three different phases: one is ferromagnetic and two are spin-singlet phases. The electron-electron interaction does not create new phases. However, it renormalizes the phase boundaries. The excitation spectrum has a gap for any value of the interlevel splitting $\Delta$ and Zeeman splitting $\Delta_{z}$. This results in a strong suppression of the electron-phonon interaction. The rate of phonon absorption by the considered quasi-2D electron system has a double-peak structure as a function of level splitting and a steplike structure as a function of Zeeman splitting.

The authors acknowledge support of the UK EPSRC.
*Electronic address: m.e.portnoi@ex.ac.uk

${ }^{1}$ R.E. Prange and S.M. Girvin, The Quantum Hall Effect (Springer, Heidelberg, 1987).

${ }^{2}$ T. Chakraborty and P. Pietilainen, The Fractional Quantum Hall Effect, 2nd ed. (Springer, New York, 1995).

${ }^{3}$ Perspectives in Quantum Hall Effects, edited by S. Das Sarma and A. Pinczuk (Wiley, New York, 1997).

${ }^{4}$ T. Chakraborty, Adv. Phys. 49, 959 (2000).

${ }^{5}$ S. Das Sarma, S. Sachdev, and L. Zheng, Phys. Rev. B 58, 4672 (1998).

${ }^{6}$ A.S. Plaut, I.V. Kukushkin, K.v. Klitzing, and K. Ploog, Phys. Rev. B 42, 5744 (1990).
${ }^{7}$ D.D. Arnone, T.P. Marlow, C.L. Foden, E.H. Linfield, D.A. Ritchie, and M. Pepper, Phys. Rev. B 56, R4340 (1997).

${ }^{8}$ V.M. Apalkov and M.E. Portnoi, cond-mat/0111382 (unpublished); V.N. Golovach and M.E. Portnoi, cond-mat/0202179 (unpublished).

${ }^{9}$ L.J. Challis and A.J. Kent, in Aspects of Semiconductor Nanostructures, edited by G. Bauer, F. Kuchar, and H. Heinrich (Springer, Heidelberg, 1992).

${ }^{10}$ C.J. Mellor, R.H. Eyles, J.E. Digby, A.J. Kent, K.A. Benedict, L.J. Challis, M. Henini, C.T. Foxon, and J.J. Harris, Phys. Rev. Lett. 74, 2339 (1995)

${ }^{11}$ U. Zeitler, A.M. Devitt, J.E. Digby, C.J. Mellor, A.J. Kent, K.A. 
Benedict, and T. Cheng, Physica B 249-251, 49 (1998).

${ }^{12}$ V.M. Apalkov and M.E. Portnoi, cond-mat/0111377 (unpublished).

${ }^{13}$ C. Kallin and B.I. Halperin, Phys. Rev. B 30, 5655 (1984).

${ }^{14}$ T. Ando, A.B. Fowler, and F. Stern, Rev. Mod. Phys. 54, 437 (1982).

${ }^{15}$ G.F. Giuliani and J.J Quinn, Phys. Rev. B 31, 6228 (1985).

${ }^{16}$ V.F. Gantmakher and Y.B. Levinson, Carrier Scattering in Metals and Semiconductors (North-Holland, Amsterdam, 1987).
${ }^{17}$ K.A. Benedict, R.K. Hills, and C.J. Mellor, Phys. Rev. B 60, 10984 (1999).

${ }^{18}$ R.J. Nicholas, R.J. Haug, K.v. Klitzing, and G. Weimann, Phys. Rev. B 37, 1294 (1988).

${ }^{19}$ B.B. Goldberg, D. Heiman, and A. Pinczuk, Phys. Rev. Lett. 63, 1102 (1989).

${ }^{20}$ A. Usher, R.J. Nicholas, J.J. Harris, and C.T. Foxon, Phys. Rev. B 41, 1129 (1990).

${ }^{21}$ A.P. Smith, A.H. MacDonald, and G. Gumbs, Phys. Rev. B 45, 8829 (1992). 